CERN-EP/98-73

May 13, 1998

Search for $\nu_{\mu} \rightarrow \nu_{\tau}$ oscillation using the $\tau$ decay modes into a single charged particle ${ }^{1)}$

The CHORUS Collaboration

E. Eskut, A. Kayis, G. Onengüt

Çukurova University, Adana, Turkey

R. van Dantzig, M. de Jong, J. Konijn, O. Melzer, R.G.C. Oldeman, C.A.F.J. van der Poel, J.W.E. Uiterwijk, J.L. Visschers

NIKHEF, Amsterdam, The Netherlands

M . Guler, M. Serin-Zeyrek, R. Sever, P. Tolun, M.T. Zeyrek

METU, Ankara, Turkey

N. Armenise, F. Cassol, M.G. Catanesi, M.T. Muciaccia, E. Radicioni, S. Simone, L. Vivolo

Università di Bari and INFN, Bari, Italy

A. Bülte, T. Patzak ${ }^{1}$, K. Winter

Humboldt Universität, Berlin, Germany ${ }^{2}$

P. Annis ${ }^{3}$, R. El Aidi, M. Gruwé ${ }^{4,5}$, C. Mommaert ${ }^{4,6}$, M. Vander Donckt ${ }^{5}$, B. Van de Vyver ${ }^{7}$, P. Vilain ${ }^{8}$, G. Wilquet ${ }^{8}$

Inter-University Institute for High Energies (ULB-VUB) Brussels, Belgium

P. Righini, B. Saitta

Università di Cagliari and INFN, Cagliari, Italy

E. Di Capua, C. Luppi, P. Zucchelli

Università di Ferrara and INFN, Ferrara, Italy

Y. Ishii, T. Kawamura, M. Kazuno, S. Ogawa, H. Shibuya

Toho University, Funabashi, Japan

J. Brunner, D. Cussans, J.P. Fabre, R. Ferreira ${ }^{9}$, W. Flegel, M. Litmaath, D. Macina ${ }^{10}$, R. Meijer Drees $1^{1}$, H. Meinhard, P. Migliozzi, E. Niu, H. Øverảs, J. Panman, I.M. Papadopoulos, E. Pesen ${ }^{12}$, F. Riccardi, S. Ricciardi, A. Rozanov ${ }^{13}$, D. Saltzberg ${ }^{14}$, P. Strolin $^{15}$, R. Tsenov ${ }^{16}$, Ch. Weinheimer ${ }^{17}$, H. Wong ${ }^{18}$

CERN, Geneva, Switzerland

J. Goldberg, K. Hoepfner ${ }^{19}$

Technion, Haifa, Israel M. Chikawa

Kinki University, Higashiosaka, Japan

E. Arik, A.A. Mailov

Bogazici University, Istanbul, Turkey

H.I. Jang, I.G. Park, J.S. Song, C.S. Yoon

Gyeongsang National University, Jinju, Korea

K. Kodama, N. Ushida

Aichi University of Education, Kariya, Japan

S. Aoki, T. Hara

Kobe University, Kobe, Japan

G. Brooijmans ${ }^{20}$, D. Favart, G. Grégoire, J. Hérin ${ }^{20}$

Université Catholique de Louvain, Louvain-la-Neuve, Belgium

A. Artamonov, P. Gorbunov, V. Khovansky, V. Shamanov

Institute for Theoretical and Experimental Physics, Moscow, Russian Federation

D. Bonekämper, D. Frekers, D. Rondeshagen, T. Wolff ${ }^{21}$

Westfälische Wilhelms-Universität, Münster, Germany ${ }^{2}$

K. Hoshino, M. Kobayashi, M. Komatsu, Y. Kotaka, T. Kozaki, M. Miyanishi, M. Nakamura, K. Nakazawa ${ }^{22}$, T. Nakano, K. Niu, K. Niwa, Y. Obayashi ${ }^{23}$, O. Sato, T. Toshito

Nagoya University, Nagoya, Japan

S. Buontempo, A. Cocco, N. D’Ambrosio, G. De Lellis, A. Ereditato, G. Fiorillo, F. Garufi, M. Messina, V. Palladino, S. Sorrentino, V. Tioukov

Università Federico II and INFN, Naples, Italy

K. Nakamura, T. Okusawa, T. Yoshida

Osaka City University, Osaka, Japan

\footnotetext{
1) This paper is dedicated to the memory of Yasushi Ishii, a bright colleague and a good friend, whose loss has caused us great sorrow
} 
A. Capone, D. De Pedis, S. Di Liberto, U. Dore, P.F. Loverre, L. Ludovici, A. Maslennikov²4, M.A. Mazzoni,

G. Piredda, R. Santacesaria, A. Satta

Università La Sapienza and INFN, Rome, Italy

E. Barbuto, A. di Bartolomeo, C. Bozza, G. Grella, G. Iovane, G. Romano, G. Rosa ${ }^{25}$

Università di Salerno and INFN, Salerno, Italy

Y. Sato, I. Tezuka

Utsunomiya University, Utsunomiya, Japan

${ }^{1}$ Now at College de France, Paris, France.

${ }^{2}$ Supported by the German Bundesministerium für Bildung und Forschung under contract numbers 05 6BU11P and $057 \mathrm{MS} 12 \mathrm{P}$.

${ }^{3}$ Supported by Regione autonoma della Sardegna, Italy.

${ }^{4}$ Now at CERN, Geneva, Switzerland.

${ }^{5}$ Fonds pour la Formation a la Recherche dans l'Industrie et dans l'Agriculture.

${ }^{6}$ Interuniversitair Instituut voor Kernwetenschappen.

${ }^{7}$ Nationaal Fonds voor Wetenschappelijk Onderzoek.

${ }^{8}$ Fonds National de la Recherche Scientifique.

${ }^{9}$ On leave of absence from LIP, Lisbon.

${ }^{10}$ Now at Université de Genève, Geneva, Switzerland.

${ }^{11}$ Now at University of Washington, Seattle, USA.

${ }^{12}$ On leave of absence from METU, Ankara, Turkey.

${ }^{13}$ Now at CPPM CNRS-IN2P3, Marseille, France.

${ }^{14}$ Now at U.C.L.A., Los Angeles, USA.

${ }^{15}$ On leave of absence from University of Naples "Federico II", Naples, Italy.

${ }^{16}$ On leave of absence from Sofia University, Bulgaria, with support from the Bogazici University, Centre for

Turkish-Balkan Physics Research and Applications.

${ }^{17}$ Now at University of Mainz, Mainz, Germany.

${ }^{18}$ Now at Academia Sinica, Taipei, Taiwan.

${ }^{19}$ Now at DESY, Hamburg, Germany.

${ }^{20}$ Institut Interuniversitaire des Sciences Nucléaires.

${ }^{21}$ Supported by a grant from Deutsche Forschungs Gemeinschaft.

${ }^{22}$ Now at Gifu University, Gifu, Japan.

${ }^{23}$ Now at Kamioka Observatory, Institute for Cosmic Ray Research, University of Tokyo, Gifu, Japan.

${ }^{24}$ CASPUR, Rome, Italy.

${ }^{25}$ Now at Università La Sapienza and INFN, Rome Italy.

Abstract

Part of the neutrino events collected in 1994-1995 by the CHORUS experiment has been analysed. A search was performed for $\nu_{\tau}$ charged current interactions followed by the $\tau$ lepton decay into a single charged negative hadron, a possible indication for $\nu_{\mu} \rightarrow \nu_{\tau}$ oscillation. A sample of 6,844 events without an identified muon has been located in the emulsion target. Within the selection criteria, no $\tau^{-}$candidate has been found. Combining this result with the one from the search for $\nu_{\tau}$ interactions using the muonic decay mode $(36,182$ events with an identified muon), a $90 \%$ C.L. limit on the mixing angle $\sin ^{2} 2 \theta_{\mu \tau}<1.8 \cdot 10^{-3}$ (at large $\Delta m^{2}$ ) has been derived.

Submitted to Physics Letters 
The CHORUS Collaboration has reported [1] a limit on $\nu_{\mu} \rightarrow \nu_{\tau}$ oscillation obtained by the analysis of a subsample of neutrino interactions with an identified $\mu^{-}$. This paper describes a similar search for $\tau^{-}$"singleprong" hadron decays. Although this channel represents a larger fraction of the $\tau$ decays (about $50 \%$ as compared to about $18 \%$ for the muonic decay [2]), the smaller efficiencies of the hadron track reconstruction and of the vertex location in the emulsion target lead to comparable sensitivities for both analyses.

The experimental set up and the characteristics of the CERN Wide Band Neutrino Beam are summarised in [1] and described in more detail in [3].

\section{The apparatus}

The "hybrid" CHORUS apparatus [3] combines a $770 \mathrm{~kg}$ nuclear emulsion target with several electronic detectors: trigger hodoscopes, a scintillating fibre tracker system, a hadron spectrometer, a lead/scintillator calorimeter and a muon spectrometer. The target consists of 4 stacks of 36 plates. Each plate has a $90 \mu \mathrm{m}$ transparent plastic base with $350 \mu \mathrm{m}$ emulsion on both sides.

The trigger consisted of a coincidence of three scintillator planes within an angular range of $250 \mathrm{mrad}$, supplemented by an anti-coincidence with a large veto plane upstream of the detector.

Since the analysis reported here differs from the previous search of $\tau^{-}$muonic decay mainly by the requirement of reconstructing hadron tracks, we briefly recall the features of the hadron spectrometer.

The hadron spectrometer contains an hexagonal shaped air-core magnet [4] located between the target region and the calorimeter. The magnet is $0.75 \mathrm{~m}$ long and its front and back faces are divided into six equilateral triangles with $1.5 \mathrm{~m}$ sides. A pulsed current through aluminium windings produces a homogeneous field of $0.12 \mathrm{~T}$ in each triangular volume, parallel to the outer sides of the triangle. The thickness of the front and back faces amounts to about $8 \%$ of a radiation length.

Three scintillating fibre tracker modules are associated with the magnet, one upstream and two downstream. Each module consists of two hexagonal planes divided into three adjacent sections. The fibre orientations in each section are such that a module always measures one coordinate in the bending plane of the magnet and one coordinate at a relative angle of 60 degrees. The $500 \mu \mathrm{m}$ diameter fibres are arranged to form 7-layer ribbons, they are read out by 18 optoelectronic chains.

For the data analysed in this paper, the tracking behind the magnet was complemented by 6 planes of streamer tubes of $1.05 \mathrm{~cm}$ wire spacing (only 4 planes in the 1994 run) with alternate vertical and horizontal orientation. The digital read out of the wire signals provides a hit resolution of $3 \mathrm{~mm}$ (rms) per plane.

The momentum resolution $\Delta p / p$ of the hadronic spectrometer for single track is well described by the quadratic combination of two terms: a constant term of $22 \%$ due to multiple scattering, mainly in the front and exit faces of the magnet, and a linear term of $3.5 \%$. $p(\mathrm{GeV} / \mathrm{c})$ reflecting the detector resolution.

\section{Data collection and event selection}

In the 1994-1995 running period, CHORUS collected about 969,000 triggers corresponding to $2.01 \cdot 10^{19}$ protons on target. Out of these triggers, 121,978 have no muon identified in the downstream detectors and have a vertex position compatible with one of the four emulsion target stacks. This sample of muonless events is referred to as the $0-\mu$ sample.

All hadron tracks reconstructed in the scintillating fibre trackers of the target region are extrapolated downstream and selected for further analysis if the following conditions are met:

- the track is associated with the interaction vertex and is detected by at least 11 out of the 12 fibre planes downstream from the target region;

- the track trajectory does not cross any of the 6 radial spokes of the air-core hexagonal magnet, which would otherwise introduce large multiple scattering errors;

- a successful momentum fit is obtained by using at least 5 of the fibre or streamer tube planes downstream from the air-core magnet. The fitted deflection corresponds to a particle of negative charge and momentum between 1 and $20 \mathrm{GeV} / \mathrm{c}$. The lower cut reduces the large amount of low energy particles from secondary interactions or $\gamma$ conversions. The upper cut is dictated by the momentum resolution at higher energy;

- the track forms an angle smaller than 0.4 rad with the beam axis. In view of the large background in the emulsion by muons originating from a nearby secondary beam, tracks at an angle smaller than $0.05 \mathrm{rad}$ from the direction of this beam are also excluded.

It should be noted that use has not been made of the energy deposition in the calorimeter to reject electrons or unidentified muons. The contribution of the $\tau^{-}$leptonic decay modes to the present data sample is taken into account in the evaluation of the sensitivity.

The number of events corresponding to this selection are given in Table 1 . Out of 45,572 0 - $\mu$ events with at least 1 selected track, 22,878 have so far been processed through the emulsion analysis described below.

\section{Scanning procedure \\ 3.1 Vertex location}

The various steps leading to the plate containing the vertex by means of fully automatized microscopes are identical to those described in [1]. They are independently applied here to each of the selected tracks in the event, whereas for the $1 \mu$ analysis they are applied to the muon track only. A track which has been found in the interface emulsion sheets is followed upstream in the target emulsion stack, using track segments reconstructed in the most upstream $100 \mu \mathrm{m}$ of each plate, until it disappears. The corresponding plate is defined as the vertex plate, since it should contain the primary neutrino vertex or the secondary (decay) vertex from which the track originates. The three most downstream plates of each stack are used to validate the matching with the 
Table 1: Current status of the $0-\mu$ event selection

\begin{tabular}{lll}
\hline \hline & 1994 & 1995 \\
\hline Protons on target & $0.8110^{19}$ & $1.2010^{19}$ \\
\hline Emulsion triggers & 422,000 & 547,000 \\
\hline $0-\mu$ events reconstructed and vertex predicted in emulsion & 50,383 & 71,595 \\
\hline Events with at least 1 selected negative track & 17,731 & 27,841 \\
\hline Events scanned so far & 8,908 & 12,365 \\
\hline Events within the fiducial volume & 5,476 & 6,920 \\
\hline Vertex located (in the 33 most upstream plates) & 3,164 & 3,680 \\
\hline
\end{tabular}

interface emulsion sheets and are not considered as possible vertex plates. The scanning results of the $0-\mu$ sample are summarised in Table 1.

The mean efficiency of this scan-back procedure is found to be $32 \%$, lower than the one achieved for muon tracks (40\% [1]). A detailed simulation of the scanning criteria shows that this difference mainly reflects the poorer quality of the hadron track predictions at the interface emulsion sheets, because of the difficulty of reconstruction inside a dense hadronic shower and the larger multiple scattering owing to the lower average momentum.

\subsection{Decay search}

Once the vertex plate is defined, automatic microscope measurements are performed to select the events potentially containing a decay topology (kink). Different algorithms have been applied, as a result of the progress in the scanning procedures and of the improving performance in speed of the scanning devices. They are described in [1] and briefly recalled here.

In the first procedure (applied to 33\% of the 19940 $\mu$ data scanned so far), the event is selected either when the scan-back track has a significant impact parameter with respect to the other predicted tracks or when the change in the scan-back track direction between the vertex plate and the exit from the emulsion corresponds to an apparent transverse momentum, $\left(p_{T}\right)$, larger than 250 $\mathrm{MeV} / \mathrm{c}$. For the selected events and for those with only one predicted track, digital images of the vertex plate are recorded and are inspected off-line for the presence of a kink. A sample of 1,050 located events was treated in this way.

The second procedure (applied to the remaining 1994 data and to the $19950-\mu$ statistics) is restricted to the search of long decay paths. In that case the vertex plate is assumed to contain the decay vertex of a charged parent produced in a more upstream plate. With this procedure only kink angles larger than 0.025 rad are detected. This method was used to analyse 5,794 located events.

For the events selected by either of these procedures, a computer assisted eye-scan is performed to assess the presence of a secondary vertex and measure accurately its topology. A $\tau^{-}$decay candidate must satisfy the following criteria:
1. the secondary vertex appears as a kink without black prongs, nuclear recoils, blobs or Auger electrons;

2. the transverse momentum of the decay hadron with respect to the parent direction is larger than 250 $\mathrm{MeV} / \mathrm{c}$ (to eliminate decays of strange particles);

3 . the kink occurs within 3 plates downstream from the neutrino interaction vertex plate. Because of the lower background, the kink search in the muonic decay channel was extended to 5 plates, with a gain in efficiency of about $8 \%$.

No $\tau^{-}$decay candidate has been found satisfying the selection criteria.

\section{Experimental check of the kink finding efficiency}

The kink finding efficiency, $\epsilon_{k i n k}$, has been evaluated by Monte Carlo simulation and experimentally checked looking at hadron interactions. For this purpose a sample of about $55 \mathrm{~m}$ of hadron tracks was scanned in emulsion. In the decay search procedure, 21 neutrino interaction events with a hadron interaction have been detected. This result is in good agreement with the expected value of $(24 \pm 2)$ by Monte Carlo simulation. Although the number of events is too low to draw quantitative conclusions, we can take this result as a qualitative check of the simulation of the automatic scanning procedure.

\section{$5 \quad$ Sensitivity and backgrounds}

In this section we discuss the expected background from known sources and, in absence of $\tau^{-}$candidates, limits on the $\nu_{\mu} \rightarrow \nu_{\tau}$ oscillation parameters are derived. Both signal efficiencies and background estimation have been evaluated from large samples of events, generated according to the relevant processes, passed through a GEANT [5] based simulation of the detector response. The output is then processed through the same reconstruction chain as used for data. The simulated tracks in emulsion are used to estimate the efficiencies of each scanning step. Apart from the kink detection efficiency, only ratios of acceptances enter the calculation of the experimental result and most of the systematic uncertainties of the simulation cancel out. 


\subsection{Background estimates}

Sources of background for the hadronic $\tau$ decay channel are:

- the prompt $\nu_{\tau}$ contamination of the beam [6]. For the present sample the expected background is $\sim 0.003$ events;

- the production of negative charmed particles from the anti-neutrino components of the beam. These events constitute a background if the primary $\mu^{+}$ or $e^{+}$remains unidentified. Taking into account the appropriate cross-sections and the branching ratios, in the present sample we expect $\sim 0.02$ events from these sources;

- the production of positive charmed mesons in charged current interactions, if the primary lepton is not identified and the charge of the charmed particle daughter is incorrectly measured. We expect $\sim 0.03$ events from this source in the present sample;

- the associated charm production both in charged and neutral current interactions, when one of the charmed particles is not detected. The cross-section for charm-anticharm pair production in neutral current interactions relative to the total charged current cross-section has been measured by the E531 experiment [7] to be $0.13_{-0.11}^{+0.31} \%$. The production rate of associated charm in charged current interactions is unknown, but an upper limit is available $(<0.12 \%$ [7]). In the present sample, the estimated background from this process represents $\lesssim 0.01$ events.

- the main potential background to the hadronic $\tau^{-}$ decays is due to so-called hadronic "white kinks", defined as 1-prong nuclear interactions with no heavily ionising tracks (black and grey tracks in emulsion terminology) and no evidence for nuclear break up (evaporation tracks, recoils, blobs or Auger electrons). Published data allowing to determine the white kink interaction cross-section are scarce $[8,9]$. The white kink cross-section was shown to account for about $7 \%$ of the total cross-section for pions and protons between 4 and $10 \mathrm{GeV}$ incident energy. In a dedicated experiment with $4 \mathrm{GeV}$ pions at KEK [8], a very steep fall-off in $p_{T}$ was observed and only 1 out of 58 observed kinks had a $p_{T}$ larger than $300 \mathrm{MeV} / \mathrm{c}$. Since the experimental information of the $p_{T}$ dependence is statistically poor at large values, a Monte Carlo simulation, based on a modified version of FLUKA [10, 11], has been performed. The results of this simulation are in good agreement with the $p_{T}$ dependence of the KEK measurement. An effective white kink mean free path in emulsion, $\lambda_{w k} \sim 22 \mathrm{~m}$, is obtained for a $p_{T}$ cut at $250 \mathrm{MeV} / \mathrm{c}$, using a pion energy spectrum as observed in the $0-\mu$ sample. The above result is compatible with the observation of 4 events with
$p_{T}>250 \mathrm{MeV} / \mathrm{c}$, all at large distance from the primary vertex, along a total path of $\sim 92 \mathrm{~m}$ of scan-back tracks, and corresponds to a background estimate of 0.5 events within 3 plates downstream from the primary vertex plate. As the statistics will increase, this background source will be better measured using the CHORUS data. It will be possible to significantly reduce, by kinematical analysis, the effect of this background on the sensitivity to a possible oscillation signal.

\subsection{Oscillation sensitivity}

In the usual approximation of a two-flavour mixing scheme, the probability of $\nu_{\tau}$ appearance in an initially pure $\nu_{\mu}$ beam can be expressed as

$$
\begin{aligned}
P_{\mu \tau}(E)= & \sin ^{2} 2 \theta_{\mu \tau} \cdot \int \Psi(E, L) \cdot \\
& \cdot \sin ^{2}\left(\frac{1.27 \cdot \Delta m_{\mu \tau}^{2}\left(e V^{2}\right) \cdot L(k m)}{E(G e V)}\right) \cdot d L
\end{aligned}
$$

where

- $E$ is the incident neutrino energy;

- $L$ is the neutrino flight length to the detector;

- $\theta_{\mu \tau}$ is the effective $\nu_{\mu}-\nu_{\tau}$ mixing angle;

- $\Delta m_{\mu \tau}^{2}$ is the difference of the squared masses of the two assumed mass eigenstates;

- $\Psi(E, L)$ is the fraction of $\nu_{\mu}$ with energy $E$ originating at a distance between $L$ and $L+d L$ from the emulsion target.

The $\tau^{-}$channels we considered in the $\nu_{\mu} \rightarrow \nu_{\tau}$ oscillation search we describe in this paper are:

1) $\tau \rightarrow \mu$,2) $\tau \rightarrow h, 3) \tau \rightarrow e$ and 4) $\tau \rightarrow \bar{\mu}$ (the $\mu$ is not identified) channels.

The expected number, $N_{\tau i}(i=1,2,3,4)$, of observed $\tau^{-}$decays into a channel of branching ratio $B R_{i}$ is then given by

$$
N_{\tau i}=B R_{i} \cdot \int \Phi_{\nu_{\mu}} \cdot P_{\mu \tau} \cdot \sigma_{\tau} \cdot A_{\tau i} \cdot \epsilon_{\tau i} \cdot d E
$$

with

- $B R_{(1 \text { or } 4)}=B R\left(\tau \rightarrow \nu_{\tau} \bar{\nu}_{\mu} \mu^{-}\right)=(17.35 \pm$ $0.10) \%[2])$.

- $\left.B R_{2}=B R\left(\tau \rightarrow \nu_{\tau} h^{-} n h^{0}\right)=(49.78 \pm 0.17) \%[2]\right) ;$

- $B R_{3}=B R\left(\tau \rightarrow \nu_{\tau} \bar{\nu}_{e} e^{-}\right)=(17.83 \pm 0.08) \%$ [2]);

- $\Phi_{\nu_{\mu}}$ the incident $\nu_{\mu}$ flux spectrum;

- $\sigma_{\tau}$ the charged current $\nu_{\tau}$ interaction cross-section;

- $A_{\tau i}$ the acceptance and reconstruction efficiency for the considered channel (up to the vertex plate location);

- $\epsilon_{\tau i}$ the corresponding efficiency of the decay search procedure;

With proper averaging (denoted by \langle\rangle ), $N_{\tau i}$ can also be written as a function of $n_{i}$ : 


$$
N_{\tau i}=B R_{i} \cdot n_{i} \cdot\left\langle P_{\mu \tau}\right\rangle \cdot \frac{\left\langle\sigma_{\tau}\right\rangle}{\left\langle\sigma_{\mu}\right\rangle} \cdot \frac{\left\langle A_{\tau i}\right\rangle}{\left\langle A_{\mu}\right\rangle} \cdot\left\langle\epsilon_{\tau i}\right\rangle
$$

where

- $n_{1}=N_{\mu}$ (the number of located charged current $\nu_{\mu}$ interactions corresponding to the considered event sample) and $n_{2}=n_{3}=n_{4}=\left(N_{\mu}\right)_{0-\mu}$ (the product of $N_{\mu}$ and the relative fraction of the $0-\mu$ sample for which the analysis has been completed);

- $\left\langle\sigma_{\mu(\tau)}\right\rangle=\int \frac{d \sigma_{\mu(\tau)}}{d E} \cdot \Phi_{\nu_{\mu}} \cdot d E$. It takes into account quasi-elastic interactions, resonance production and deep inelastic interactions $\left(\sigma\left(\frac{\left\langle\sigma_{\tau}\right\rangle}{\left\langle\sigma_{\mu}\right\rangle}\right)_{s y s t} \sim 7 \%\right)$;

- $\left\langle A_{\mu(\tau i)}\right\rangle=\int \frac{d \sigma_{\mu(\tau)}}{d E} \cdot A_{\mu(\tau i)} \cdot \Phi_{\nu_{\mu}} \cdot d E$ $\left(\sigma\left(\frac{\left\langle A_{\tau i}\right\rangle}{\left\langle A_{\mu}\right\rangle}\right)_{\text {syst }} \sim 7 \%\right)$;

- $\left\langle\epsilon_{\tau i}\right\rangle$ is the average efficiency of the decay search procedure for the accepted events $\left(\sigma\left(\left\langle\epsilon_{\tau i}\right\rangle\right)_{\text {syst }} \sim\right.$ $10 \%)$;

To allow an easy combination of the results from the $1-\mu$ and $0-\mu$ event samples, it is useful to define the "equivalent number of muonic events" of the $0-\mu$ sample by

$$
N_{\mu}^{e q}=\left(N_{\mu}\right)_{0-\mu} \cdot \sum_{i=2}^{4} \frac{\left\langle A_{\tau i}\right\rangle}{\left\langle A_{\tau \mu}\right\rangle} \cdot \frac{\left\langle\epsilon_{\tau i}\right\rangle}{\left\langle\epsilon_{\tau \mu}\right\rangle} \cdot \frac{B R_{i}}{B R_{\mu}}
$$

For large $\Delta m_{\mu \tau}^{2}$ values, the oscillation probability reduces to

$$
P_{\mu \tau}=\frac{1}{2} \cdot \sin ^{2} 2 \theta_{\mu \tau}
$$

and the $90 \%$ C.L. upper limit on the mixing parameter simplifies into

$$
\sin ^{2} 2 \theta_{\mu \tau} \leq 2 \cdot \frac{2.38 \cdot r_{\sigma} \cdot r_{A}}{B R_{\mu} \cdot\left\langle\epsilon_{\tau \mu}\right\rangle \cdot\left[N_{\mu}+N_{\mu}^{e q}\right]}
$$

where $r_{\sigma}=\left\langle\sigma_{\mu}\right\rangle /\left\langle\sigma_{\tau}\right\rangle$ and $r_{A}=\left\langle A_{\mu}\right\rangle /\left\langle A_{\tau \mu}\right\rangle$.

In the above formula, the numerical factor 2.38 takes into account the total systematic error (17\%) following the prescription given in [12]. The systematic error is mainly due to the reliability of the Montecarlo simulation of the scanning procedures.

The estimated values of the quantities appearing in this expression are given in Table 2. No statistical errors are quoted since they are much smaller than the systematic uncertainty.

Using the $0-\mu$ sample alone, they lead to the $90 \%$ C.L. limit (at large $\Delta m^{2}$ )

$$
P_{\mu \tau} \leq 2.3 \cdot 10^{-3}
$$

and

$$
\sin ^{2} 2 \theta_{\mu \tau} \leq 4.6 \cdot 10^{-3}
$$

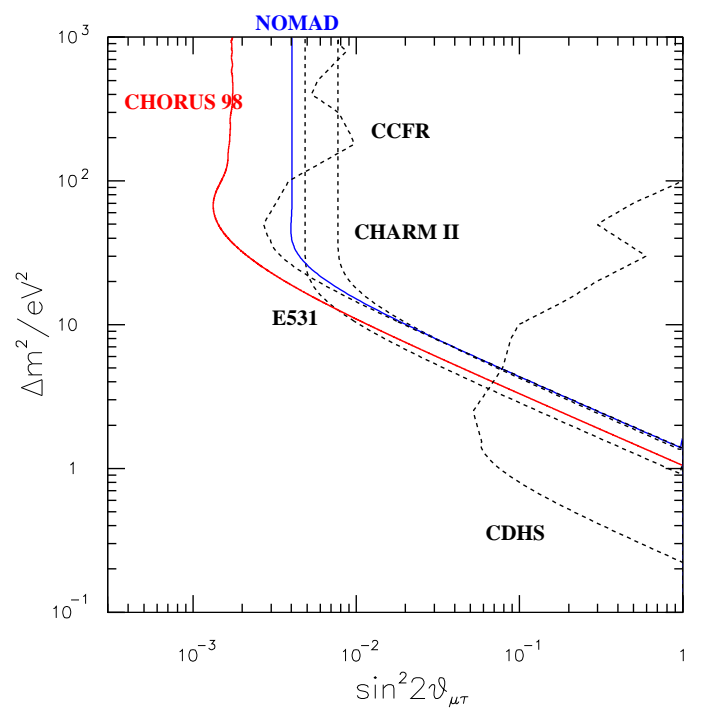

Figure 1: Present result compared with the recent NOMAD result [13] (full line) and the previous limits (dotted lines).

Adding the 1- $\mu$ sample, with an updated statistics with respect to the one described in [1] $(36,182$ events in total with an identified $\mu^{-}$), the $90 \%$ C.L. limit on the mixing parameter becomes (at large $\Delta m^{2}$ )

$$
\sin ^{2} 2 \theta_{\mu \tau} \leq 1.8 \cdot 10^{-3}
$$

A more detailed calculation, taking into account the energy dependence of the different factors, is used to derive the $90 \%$ C.L. excluded region in the $\left(\sin ^{2} 2 \theta_{\mu \tau}\right.$, $\Delta m_{\mu \tau}^{2}$ ) parameter space represented in Figure 1. Maximum mixing between $\nu_{\mu}$ and $\nu_{\tau}$ is excluded at $90 \%$ C.L. if $\Delta m_{\mu \tau}^{2}>1.1 \mathrm{eV}^{2}$.

\section{Conclusions}

The emulsion scanning methods, previously developed to search for a $\tau^{-}$signal in the neutrino interactions with an identified $\mu^{-}$, have been extended to analyse a fraction of the $0-\mu$ sample collected by CHORUS in 1994-1995. No $\tau^{-}$decay candidate has been found, leading to a more stringent $90 \%$ C.L. upper limit on the $\nu_{\mu} \rightarrow \nu_{\tau}$ oscillation probability $\left(P_{\mu \tau} \leq 0.9 \cdot 10^{-3}\right.$ and $\sin ^{2} 2 \theta_{\mu \tau} \leq 1.8 \cdot 10^{-3}$ at large $\left.\Delta m^{2}\right)$. This negative result is compatible with an estimated background of less than one event, mainly from "white kink" secondary interactions. With the large increase in statistics expected from the ongoing analysis of the 1996-97 data, a direct measurement of this background process will be possible and kinematical cuts are planned for its reduction. Furthermore, a second phase of the analysis (with better efficiencies, larger statistics and faster automatic emulsion scanning) has started with the aim of reaching the design sensitivity $\left(\sin ^{2} 2 \theta_{\mu \tau} \leq 2 \cdot 10^{-4}\right)$. 
Table 2: Quantities used in the estimation of the sensitivity

\begin{tabular}{lll}
\hline \hline & 1994 & 1995 \\
\hline$N_{\mu}$ & 18286 & 17896 \\
$r_{\sigma}$ & 1.89 & 1.89 \\
$r_{A}$ & 0.93 & 0.93 \\
$\left\langle A_{\tau \mu}\right\rangle$ & 0.39 & 0.39 \\
$\left\langle A_{\tau h}\right\rangle$ & 0.17 & 0.17 \\
$\left\langle A_{\tau e}\right\rangle$ & 0.093 & 0.093 \\
$\left\langle A_{\tau \bar{\mu}}\right\rangle$ & 0.026 & 0.026 \\
$\left\langle\epsilon_{\tau \mu}\right\rangle$ & 0.53 & 0.35 \\
$\left\langle\epsilon_{\tau h}\right\rangle$ & 0.24 & 0.25 \\
$\left\langle\epsilon_{\tau e}\right\rangle$ & 0.12 & 0.13 \\
$\left\langle\epsilon_{\tau \bar{\mu}}\right\rangle$ & 0.22 & 0.23 \\
$N_{\mu}^{e q}$ & 11987 & 12119 \\
$\left(N_{\mu}\right)_{0-\mu}$ & 17107 & 11632 \\
\hline
\end{tabular}

\section{Acknowledgements}

We gratefully acknowledge the help and support of our numerous technical collaborators who contributed to the detector construction, operation, emulsion pouring, development and scanning. We thank the neutrino beam staff for their competent assistance in ensuring the excellent performance of the facility. The accumulation of a large data sample in this experiment has been made possible also thanks to the efforts of the crew operating the CERN PS and SPS. The general technical support from CERN EP and IT divisions is warmly acknowledged.

The experiment has been made possible by grants from: the Institut Interuniversitaire des Sciences Nucléaires and the Interuniversitair Instituut voor Kernwetenschappen (Belgium), the Israel Science foundation (grant 328/94) and the Technion Vice President Fund for the Promotion of Research (Israel), CERN (Geneva, Switzerland), the German Bundesministerium für Bildung und Forschung (Germany), the Institute of Theoretical and Experimental Physics (Moscow, Russia), the Istituto Nazionale di Fisica Nucleare (Italy), the Japan Private School Promotion Foundation and Japan Society for the Promotion of Science (Japan), the Korea Science and Engineering Foundation and the Ministry of Education through Research Fund (BSRI-97-2407) (Republic of Korea), the Foundation for Fundamental Research on Matter FOM and the National Scientific Research Organization NWO (The Netherlands) and the Scientific and Technical Research Council of Turkey (Turkey).

We gratefully acknowledge their support.

\section{References}

[1] E. Eskut et al., CHORUS Collaboration, Phys. Lett. B 424 (1998) 202.

[2] Particle Data Group, Phys. Rev. D54 1 (1996).

[3] E. Eskut et al., CHORUS Collaboration, Nucl. Instr. and Meth. A401 7 (1997).
[4] F. Bergsma et al., Nucl. Instr. and Meth. A357 253 (1995).

[5] GEANT 3.21, CERN Program Library Long Writeup W5013.

[6] B. Van der Vyver, Nucl. Instr. and Meth. A385 91 (1997).

[7] N. Ushida et al., E531 Collaboration, Phys. Lett. B206 375 (1988).

[8] K. Kodama et al., FERMILAB P803 Proposal (1993), Appendix D.

[9] F. Baldassarre et al., Nuovo Cimento XXI, 3 (1961) 459.

[10] A. Fassò, A. Ferrari, J. Ranft and P.R. Sala, "New developments in FLUKA modelling of hadronic and EM interactions", in Proceedings of the 3rd workshop on "Simulating Accelerator Radiation Environment", SARE-3, KEK-Tsukuba, May 7-9 1997, H. Hirayama ed., KEK report Proceedings 97-5, p. 32 (1997).

[11] A. Ferrari, T. Rancati and P.R. Sala, "FLUKA applications in high energy problems: from LHC to ICARUS and atmospheric showers", in Proceedings of the 3rd workshop on "Simulating Accelerator Radiation Environment", SARE-3, KEKTsukuba, May 7-9 1997, H. Hirayama ed., KEK report Proceedings 97-5, p. 165 (1997).

[12] R.D. Cousins and V.L. Highland, Nucl. Instr. and Meth. A320 331 (1992).

[13] J. Altegoer et al., NOMAD Collaboration, CERNEP/98-57. 